TH-138: Phase II Randomized Trial of Carboplatin + Pemetrexed + Bevacizumab, With or Without Atezolizumab, in Patients With Stage IV Nonsquamous NSCLC Who Harbor a Sensitizing EGFR Mutation or Have Never Smoked

Principal Investigator: Joseph Treat, MD

Condition: Stage IV nonsquamous non-small cell lung cancer Institution: Fox Chase Cancer Center

Patients with non-small cell lung cancer (NSCLC) who harbor an EGFR mutation or are never-smokers generally do not benefit from singleagent immunotherapy. Subgroup analyses from the recent phase III IMpower150 trial suggested that immunotherapy-chemotherapy with vascular endothelial growth factor inhibition may overcome immunotherapy resistance in patients with EGFR-mutated tumors. Further prospective research is needed to explore the checkpoint inhibitorVEGF inhibitor combination. None of the checkpoint inhibitors are currently approved in first-line treatment for patients with EGFR mutations after failure of tyrosine kinase inhibitor therapy. This phase II study proposes to exclusively examine a population of patients with stage IV nonsquamous NSCLC who either harbor a sensitizing EGFR exon 19 or 21 mutation or are never-smoker wild-types to determine whether adding the PD-L1 inhibitor atezolizumab in combination with pemetrexed/carboplatin/bevacizumab can improve outcomes. Neversmoker wild-type is defined as smoking $<100$ cigarettes in a lifetime and without any EGFR mutation or ALK or ROS1 rearrangement.

\section{Primary Objective:}

- Compare progression-free survival (PFS) in patients receiving atezolizumab in addition to pemetrexed/carboplatin/bevacizumab (Arm A) versus those receiving pemetrexed/carboplatin/bevacizumab without atezolizumab (Arm B)

\section{Secondary Objectives:}

- Perform a safety analysis in all treated subjects

- Compare the overall response rate of Arm A versus Arm B

- Compare the duration of response of Arm A versus Arm B

- Compare the time to response of Arm A versus Arm B

- Compare PFS and overall survival of Arm A versus Arm B in the subset of patients with tumors with a known EGFR exon 19 or 21 mutation

Contact: Joseph Treat, MD • 215-214-4297• Joseph.Treat2@fccc.edu

ClinicalTrials.gov Identifier: NCT03786692
The NCCN Oncology Research Program (ORP) strives to improve the quality of life for patients and reduce cancer-related deaths by advancing cancer therapies through research. Since the program's establishment in 1999, the NCCN ORP has brought millions of dollars in research grants to investigators at NCCN Member Institutions. Research grants are provided to NCCN through collaborations with pharmaceutical and biotechnology companies; these grants are in turn used to support scientifically meritorious cancer research efforts.

NCCN ORP studies typically explore new avenues of clinical investigation and seek answers to important cancer-related questions. All studies are approved and funded through a scientific peer-review process and are overseen by the ORP.

This feature highlights an NCCN study funded through the grant mechanism.

doi: $10.6004 /$ jnccn.2020.0034

For more information on specific trials, including patient selection criteria, use the contact information listed with each study.

For more information on the NCCN ORP, including a complete detailing of the clinical studies currently underway at NCCN Member Institutions, go to www. NCCN.org/clinical_trials/clinicians.asp. 